POS $\quad$ PROCEEDINGS

\title{
WIMPs annihilations in Pop III stars
}

\author{
Marco Taoso* $\dagger$ \\ INFN, Sezione di Padova, via Marzolo 8,Padova, 35131, Italy and \\ Institut d'Astrophysique de Paris, UMR 7095-CNRS, Université Pierre et Marie Curie, 98 bis \\ Boulevard Arago 75014, Paris, France \\ E-mail: taoso@pd.infn.it
}

\section{Gianfranco Bertone}

Institut d'Astrophysique de Paris, UMR 7095-CNRS, Université Pierre et Marie Curie, 98 bis

Boulevard Arago 75014, Paris, France

E-mail: bertonediap.fr

\section{Georges Meynet}

Geneva Observatory, University of Geneva

Maillettes 51, 1290 Sauverny, Switzerland.

E-mail: georges.meynet@obs.unige.ch

\section{Sylvia Ekström}

Geneva Observatory, University of Geneva

Maillettes 51, 1290 Sauverny, Switzerland.

E-mail: Sylvia.Ekstromeobs.unige.ch

We study the impact of the capture and annihilation of Weakly Interacting Massive Particles (WIMPs) on the evolution of Pop III stars. With a suitable modification of the Geneva stellar evolution code, we study the evolution of 20,60 and $200 \mathrm{M}_{\odot}$ stars in Dark Matter haloes with densities between $10^{8}$ and $10^{11} \mathrm{GeV} / \mathrm{cm}^{3}$ during the core $\mathrm{H}$-burning phase, and, for selected cases, until the end of the core He-burning phase. We find that for WIMP densities higher than $5.310^{10}\left(\sigma_{p}^{S D} / 10^{-38} \mathrm{~cm}^{2}\right)^{-1} \mathrm{GeV} \mathrm{cm}^{-3}$ the core H-burning lifetime of these stars exceeds the age of the Universe, and stars are sustained only by WIMP annihilations. We determine the observational properties of these "frozen' objects and show that they can be searched for in the local Universe thanks to their anomalous mass-radius relation, which should allow unambiguous discrimination from normal stars.

Identification of dark matter 2008

August 18-22, 2008

Stockholm, Sweden

\footnotetext{
*Speaker.

${ }^{\dagger}$ We thank the organisers of the idm08 conference for the stimulating atmosphere and for partial support covering local expenses.
} 


\section{Introduction}

Among the many Dark Matter (DM) candidates proposed in literature, Weakly Interacting Massive Particles (WIMPs) are the most studied. Mainly, this is due to their deep connection with well motivated extensions of the Standard Model and to the strength of their annihilation and scattering cross sections, which make them naturally inherit the right cosmological abundance and open the possibility to detect them by current or upcoming direct and indirect experiments (See Ref.[1] for recent reviews on particle Dark Matter).

For sizeable scattering cross section off baryons, WIMPs can in addition produce macroscopic effects on astrophysical objects. In fact, DM particles crossing a star could be captured, accumulate at the center and annihilate, providing therefore a source of energy which can eventually modify the star evolution and properties. However, the more and more constraining bounds on the DM-baryon interaction coming from direct detection experiments force the searches of these effects to focus on objects placed in regions of high Dark matter densities, like the galactic center. This region has been indeed the target of many recent studies focusing on the consequences of DM capture in stars, in particular for the case of White Dwarfs [2], compact objects [3] and main sequence stars [4,5].

Alternatively, it has been recently noticed that DM annihilations can lead dramatic consequences in halos hosting the formation of first stars, also called Pop III stars. Pop III stars are thought to form from gas collapsing at the center of $10^{6}-10^{8} M_{\odot}$ DM halos at redshift $z \lesssim 10-30$. During the infall of gas at the center of the halo, a steepening of the Dark Matter profile is produced by adiabatic contraction and consequently high Dark Matter densities can be reached in the central regions. Spolyar, Freese and Gondolo [6] have first investigated the consequences of DM annihilations during the formation of the first proto-stars, showing that the energy released by WIMP annihilations in these mini-halos may exceed any cooling mechanism, thus leading to a new phase of stellar evolution (see also Ref. [7]). These effects could significantly delay the star formation and modify the its final mass function [8]. It was subsequently shown in Refs. [9, 10], that the annihilation of DM particles captured at the center of the star, due to scattering off the stellar nuclei, can lead to an energy injection that overwhelms nuclear reactions. Under these circumstances, the core H-burning phase of Pop III stars, in DM halos of density of $10^{11} \mathrm{GeV} \mathrm{cm}^{-3}$, is substantially prolonged, especially for small mass stars $\left(M_{*}<40 M_{\odot}\right)$ [11]. Detailed studies performed with stellar codes have indeed found that above a critical DM density, the annihilation of WIMPs captured by Pop. III stars can block the evolution of these objects, prolonging their lifetime as long as the DM density remains higher than this critical value $[12,13]$.

Here we present the result of the analysis in Ref.[12], obtained with a with a suitable modification of the Geneva stellar evolutiona code [14].

\section{WIMPs capture and annihilations.}

The capture rate is computed, following Ref. [15], as

$$
C=4 \pi \int_{0}^{R_{*}} d r r^{2} \frac{d C(r)}{d V}
$$



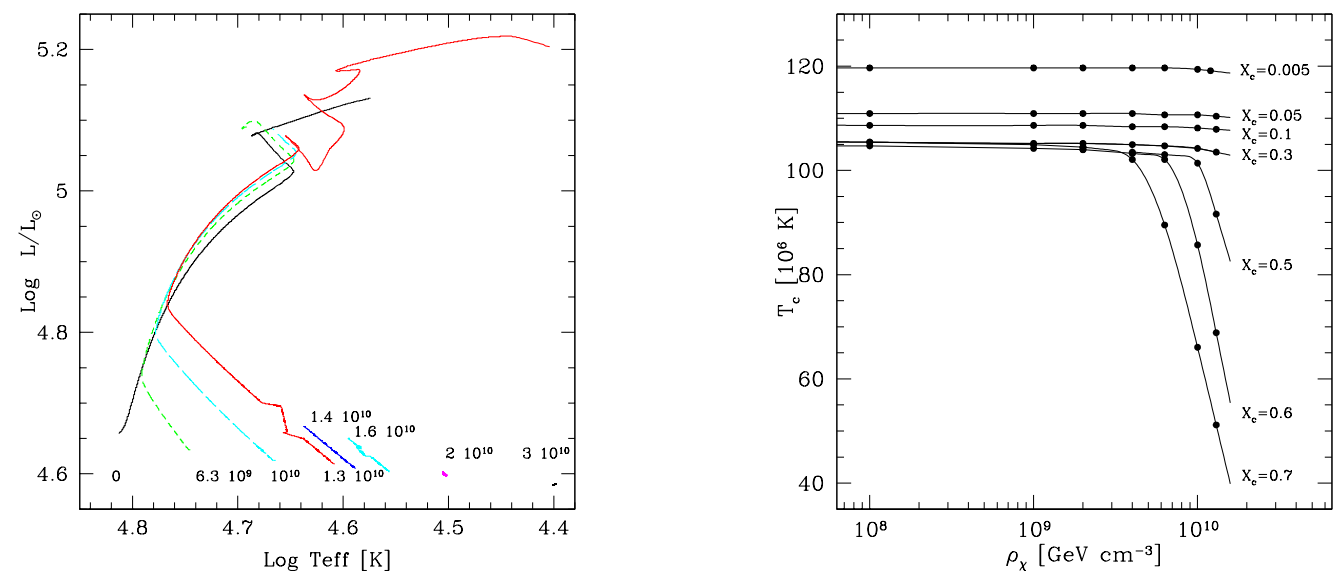

Figure 1: Left: evolutionary tracks of a Pop III $20 \mathrm{M}_{\odot}$ star for different WIMP densities (labels in units of $\mathrm{GeV} \mathrm{cm}^{-3}$ ). We have adopted a WIMP model with $m_{\chi}=100 \mathrm{GeV}$ and $\sigma_{p}^{S D}=10^{-38} \mathrm{~cm}^{2}$. Right: core temperature as a function of the DM density at different stages of the core H-burning phase. $X_{c}$ denotes the mass fraction of hydrogen at the centre ( $X_{c}=0.76$ at the beginning of the core H-burning phase). WIMP parameters and star model as in right panel.

with

$$
\begin{aligned}
& \frac{d C(r)}{d V}=\left(\frac{6}{\pi}\right)^{1 / 2} \sigma_{\chi, N} \frac{\rho_{i}(r)}{M_{i}} \frac{\rho_{\chi}}{m_{\chi}} \frac{v^{2}(r)}{\bar{v}^{2}} \frac{\bar{v}}{2 \eta A^{2}} \\
& \times\left\{\left(A_{+} A_{-} \frac{1}{2}\right)\left[\chi(-\eta, \eta)-\chi\left(A_{-}, A_{+}\right)\right]+\frac{1}{2} A_{+} e^{-A_{-}^{2}}-\frac{1}{2} A_{-} e^{-A_{+}^{2}}-\frac{1}{2} \eta e^{-\eta^{2}}\right\} \\
& A^{2}=\frac{3 v^{2}(r) \mu}{2 \bar{v}^{2} \mu_{-}^{2}}, \quad A_{ \pm}=A \pm \eta, \quad \eta^{2}=\frac{3 v_{*}^{2}}{2 \bar{v}^{2}} \\
& \chi(a, b)=\frac{\sqrt{\pi}}{2}[\operatorname{Erf}(b)-\operatorname{Erf}(a)]=\int_{a}^{b} d y e^{-y^{2}}, \quad \mu_{-}=\left(\mu_{i}-1\right) / 2, \quad \mu_{i}=m_{\chi} / M_{i}
\end{aligned}
$$

where $\rho_{i}(r)$ is the mass density profile of a given chemical element in the interior of the star and $M_{i}$ refers to its atomic mass, while $\rho_{\chi}, m_{\chi}$ and $\bar{v}$ are respectively the WIMP mass and the WIMP density and velocity dispersion at the star position. The velocity of the star with respect to an observer, labeled as $v_{*}$, is assumed to be equal to $\bar{v}$, giving therefore $\eta=\sqrt{3 / 2}$, and we take $\bar{v}=$ $10 \mathrm{Km} \mathrm{s}^{-1}$, the DM virial velocity in an halo of $10^{5}-10^{6} M_{\odot}$ at $\mathrm{z}=20$. The radial escape velocity profile depends on $M(r)$, i.e. the mass enclosed within a radius $r, v^{2}(r)=2 \int_{r}^{\infty} G M\left(r^{\prime}\right) / r^{\prime 2} d r^{\prime}$.

As for the DM density, semi-analytic computations of the adiabatic contraction of DM halos [6], in agreement with the results extrapolated from simulations of first star formation [17], suggest DM densities of order $10^{12} \mathrm{GeV} \mathrm{cm}^{-3}$ or even higher.

The WIMP scattering cross section off nuclei, $\sigma_{\chi, N}$ is constrained by direct detection experiments and for a WIMP mass of $100 \mathrm{GeV}$ the current upper limits are $\sigma_{S I}=10^{-43} \mathrm{~cm}^{2}$ [18] and $\sigma_{S D}=10^{-38} \mathrm{~cm}^{2}$ [19] respectively for spin-independent and spin-dependent WIMP interactions off a proton. We have adopted these reference values in the analysis, but the capture rate can be 

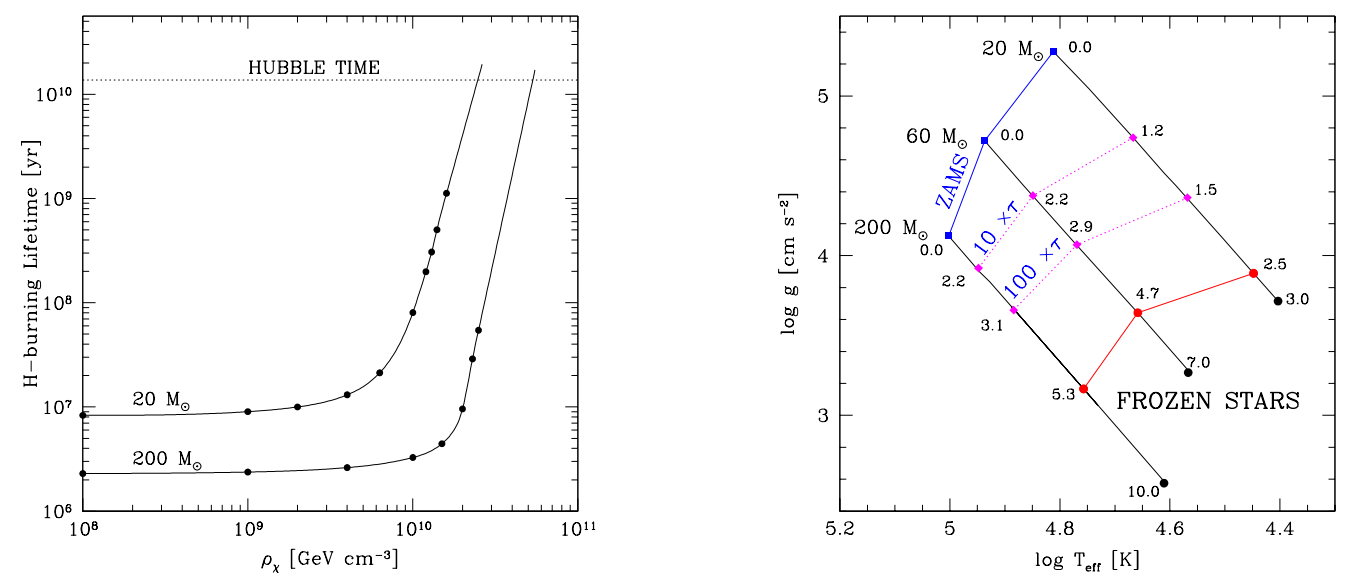

Figure 2: Left: Variation of the core H-burning lifetime as a function of the WIMP densities for the Pop III 20 and $200 \mathrm{M}_{\odot}$ models. Right: ZAMS positions of 20, 60 and $200 \mathrm{M}_{\odot}$ Pop. III stars in the g vs. $T_{\text {eff }}$ plane for different $\mathrm{DM}$ densities (labels in units of $\mathrm{GeV} \mathrm{cm}^{-3}$ ). Big red circles correspond to the critical WIMP density (see text). The lines labeled as $10 \tau$ and $100 \tau$ correspond to models with lifetime prolonged by 10 and 100 times with respect to the case without WIMPs. WIMPs parameters as in Fig. 1.

easily rescaled for other scattering cross sections by using Eq. 2.3. The spin-independent interactions with nucleons inside nuclei add up coherently giving an enhancement factor $A^{4}$ with respect to the interaction with a single nucleon: $\sigma_{\chi, N}^{S I}=A^{4} \sigma_{\chi, p}$, where $A$ is the mass number.

Once captured, WIMPs get redistributed in the interior of the star reaching, in a characteristic time $\tau_{t h}$, a thermal distribution [20]:

$$
n_{\chi}(r)=n_{0} e^{\frac{-r^{2}}{r_{w}^{2}}} \text { with } r_{\chi}=\sqrt{\frac{3 k T_{c}}{2 \pi G \rho_{c} m_{\chi}}}
$$

with $T_{c}$ and $\rho_{c}$ referring to the core temperature and density. The distribution results quite concentrated toward the center of the star: e.g. for a $20 M_{\odot}$ star immersed in a WIMP density of $\rho_{\chi}=10^{9} \mathrm{GeV} \mathrm{cm}^{-3}$ at the beginning of the core H-burning phase we obtain $r_{\chi}=2 \times 10^{9} \mathrm{~cm}$, a value much lower than the radius of the star, $R_{*}=10^{11} \mathrm{~cm}$.

An upper limit for the transient $\tau_{t h}$ can be obtained as follows: $\tau_{t h}=\left(m_{\chi} / M_{H}\right) /\left(\sigma_{S D} \bar{n}_{H} \bar{v}\right)$ where $\overline{n_{H}}$ is the average density on the star.

The WIMPs luminosity is simply $L_{\chi}(r)=4 \pi(\sigma v) m_{\chi} c^{2} n_{\chi}^{2}(r)$. For the annihilation cross section times relative velocity $(\sigma v)$, we assume the value $3 \times 10^{-26} \mathrm{~cm}^{2}$, as appropriate for a thermal WIMP, but note that the total WIMP luminosity at equilibrium does not depend on this quantity. After a time $\tau_{\chi}=\sqrt{C(\sigma v) \pi^{-3 / 2} r_{\chi}^{-3}}$ an equilibrium between capture and annihilation is established, and this incidentally allows to determine the normalization constant $n_{0}$ above.

We have checked that the two transients $\tau_{\chi}$ and $\tau_{t h}$ remain much smaller, during the evolution of the star, than the Kelvin-Helmotz timescale, $\tau_{K H}$ and the timescale needed for the nuclear reactions to burn an hydrogen fraction $\Delta X_{c}=0.002$ of the convective core (see Ref.[12] for more details). This argument justifies the assumption of equilibrium between capture and annihilation and the use of the radial distribution in Eq. 2.3. 


\section{Results}

We have implemented the effects of WIMPs annihilation in the Geneva stellar evolution code and followed the evolution of a $20 M_{\odot}$ and $60 M_{\odot}$ and $200 M_{\odot}$ stars for different DM densities. In Fig. 2 we show the evolutionary tracks for the $20 M_{\odot}$ model, and for comparison (black line) the case of a standard Pop III star without WIMPs. For DM densities smaller than $10^{9} \mathrm{GeV} \mathrm{cm}^{-3}$ the evolutionary tracks closely follow that of a normal star and they are not shown for simplicity. The position of the star at the beginning of the core H-burning phase (zero-age main sequence, or ZAMS) is obtained when, after a short transient, the luminosity produced at the center of the star equals the total luminosity and the star settles down in a stationary regime. For increasing DM densities the WIMPs luminosity produced at the center overwhelms the luminosity from nuclear reactions and makes the star inflate, producing therefore a substantial decrease of the effective temperature and a moderate decrease of the star luminosity at the ZAMS position, with respect to the standard scenario. For $\rho_{\chi}=10^{10} \mathrm{GeV} \mathrm{cm}^{-3}$, the energy produced by WIMPs present in the star at a given time, estimated as $E_{\chi} \simeq L_{\chi} \tau_{K H}$, is, at the ZAMS, $\sim 0.8$ times the gravitational potential energy of the star, and the star therefore starts to contract. In this phase, the core temperature, and consequently also the nuclear reactions, increase. When the latter become comparable with the WIMPs luminosity, the standard situation is recovered and the evolutionary track joins the classical tracks of a star without WIMPs. An important difference from standard evolution is that in the first phase, the nuclear reactions are slowed down and therefore the core $\mathrm{H}$-burning lifetime is prolonged. For Dark Matter densities $\rho_{\chi} \leq 1.610^{10} \mathrm{GeV} \mathrm{cm}^{-3}$, the picture is qualitatively the same, and for these models we only show in Fig. 2the first phases of the evolution. In Fig. 2, we also show the core temperature as a function of the DM density, at different stages of the core H-burning phase. At high DM densities hydrogen burns at much lower core temperatures than in the usual scenario, till a certain mass fraction is reached, e.g. $X_{c}=0.3$ for $\rho_{\chi}=10^{10} \mathrm{GeV} \mathrm{cm}^{-3}$, and the standard evolutionary track is joined. For increasing DM densities the nuclear reaction rate is more and more delayed till the contraction of the star is inhibited, due to the high DM energy accumulated, and the evolution is frozen. In Fig.2 for $\rho_{\chi}=2 \cdot 10^{10} \mathrm{GeV} \mathrm{cm}^{-3}$ and $\rho_{\chi}=$ $3 \cdot 10^{10} \mathrm{GeV} \mathrm{cm}{ }^{-3}$ the stars seems to remain indefinitely at the ZAMS position. In Fig. 2 we show the core H-burning lifetime as a function of the DM density. In the case of a $20 M_{\odot}$ model, for $\rho \leq 10^{9} \mathrm{GeV} \mathrm{cm}^{-3}$ the core H-burning phase is prolonged by less then $10 \%$ but the delay increases rapidly for higher DM densities. Extrapolating the curve we determine a critical density, $\rho_{c}=2.5$. $10^{10} \mathrm{GeV} \mathrm{cm}^{-3}$, beyond which the core H-burning lifetime is longer then the age of the Universe. All the calculations have been repeated for the $60 M_{\odot}$ and $200 M_{\odot}$ models and we find that in all the cases the star evolution is stopped for DM densities higher than $5.3 \cdot 10^{10}\left(\frac{\sigma_{p}^{S D}}{10^{-38} \mathrm{~cm}^{2}}\right)^{-1} \mathrm{GeV} \mathrm{cm}^{-3}$. We have also verified that the results weakly depend on the WIMP mass, e.g. the core H-lifetime is modified by a factor $0.2 \%$ and $5 \%$ respectively for $m_{\chi}=10 \mathrm{GeV}$ and $m_{\chi}=100 \mathrm{GeV}$, if $\rho_{\chi}=$ $10^{10} \mathrm{GeV} \mathrm{cm}^{-3}$.

It is remarkable that under these circumstances, frozen Pop III stars can survive until the present epoch, and can be searched for as an anomalous stellar population. In Fig.2 we show the effective temperature and gravity acceleration at the surface of these frozen Pop III stars, kept in the H-burning phase, for different DM densities. Frozen stars would thus appear much bigger and with much lower surface temperatures with respect to normal stars with the same mass and 
metallicity. Our results are qualitatively consistent with the preliminary estimates in $[9,10]$ and the analysis in $[11,13]$. However, for a given DM density, we obtain a somewhat longer core H-burning lifetime with respect to [11], possibly due to their use of an approximated expression for the capture rate. We have also followed, for selected models, the evolution during the core He-burning phase. During this evolutionary stage, the Dark Matter luminosity is lower than the nuclear reaction luminosity, therefore the impact of DM annihilations is found to be rather weak. For the $20 M_{\odot}$ model and for $\rho_{\chi}=1.6 \cdot 10^{10} \mathrm{GeV} \mathrm{cm}^{-3}$ the He-lifetime is prolonged by a factor 1.2 , rather than a factor 37 found for the H-burning phase for the same DM density.

In conclusion, we have adapted a stellar evolution code to the study the evolution of Pop. III stars in presence of WIMPs. We have shown that above a critical DM density, the annihilation of WIMPs captured by Pop. III stars can dramatically alter the evolution of these objects, and prolong their lifetime beyond the age of the Universe. We have determined the properties of these 'frozen' stars, and determined the observational properties that may allow to discriminate these objects from ordinary stars.

\section{References}

[1] G. Bertone, D. Hooper and J. Silk, Phys. Rep. 405 (2005) 279; L. Bergstrom, Rept. Prog. Phys. 63, 793 (2000).

[2] I.V. Moskalenko and L.L. Wai, Astrophys. J. 659:L29-L32, 2007 [arXiv:astro-ph/0702654].

[3] G. Bertone and M. Fairbairn, Phys. Rev. D 77 (2008) 043515 [arXiv:0709.1485 [astro-ph]].

[4] M. Fairbairn, P. Scott and J. Edsjo, Phys. Rev. D 77 (2008) 047301.

[5] P.Scott, M.Fairbairn and J.Edsjo, [arXiv:0809.1871].

[6] D. Spolyar, K. Freese and P. Gondolo, Phys. Rev. Lett. 100 (2008) 051101.

[7] K. Freese, P. Bodenheimer, D. Spolyar and P. Gondolo, 2008 [arXiv:0806.0617].

[8] Talk by E.Ripamonti at the idm08 conference.

[9] F. Iocco, Astrophys. J. 677 (2008) L1.

[10] K. Freese, D. Spolyar and A. Aguirre, 2008 [arXiv:0802.1724].

[11] F. Iocco, A. Bressan, E. Ripamonti, R. Schneider, A. Ferrara and P. Marigo, 2008 [arXiv:0805.4016].

[12] M.Taoso, G.Bertone, G.Meynet and S.Ekstrom, 2008 [arXiv:0806.2681].

[13] S. Yoon, F. Iocco and S. Akiyama 2008 [arXiv:0806.2662].

[14] S. Ekström, G. Meynet, A. Maeder and F. Barblan, Astronomy and Astrophysics 478 (2008) 467.

[15] A. Gould, ApJ 567 (1987) 532.

[16] K. Freese, P. Gondolo, J.A. Sellwood and D. Spolyar, [arXiv:0805.3540].

[17] T. Abel, G.L. Bryan and M.L. Norman, Science 295 (2002) 93.

[18] Z. Ahmed et al. [CDMS Collaboration], arXiv:0802.3530 [astro-ph]; J. Angle et al. [XENON Collaboration], Phys. Rev. Lett. 100 (2008) 021303 [arXiv:0706.0039 [astro-ph]].

[19] J. Angle et al., arXiv:0805.2939 [astro-ph]; E. Behnke et al. [COUPP Collaboration], Science 319 (2008) 933 [arXiv:0804.2886 [astro-ph]].

[20] K.Griest and D.Seckel, Nucl. Phys. B 296 (1987) 681. 